\title{
Relation of alcohol to Helicobacter pylori infection: A cross sectional study
}

\author{
Rakshitha HB ${ }^{1}$, Amita K ${ }^{2, *}$, Mangala Gouri ${ }^{3}$, Avinash Balekuduru ${ }^{4}$ \\ ${ }^{\mathbf{1}}$ Assistant Professor, ${ }^{2}$ Professor, Dept. of Pathology, Adichunchanagiri Institute of Medical Sciences, Karnataka, ${ }^{3}$ Professor, \\ ${ }^{4}$ Associate Professor, Dept. of Pathology, M.S. Ramaiah Memorial Hospital, Bangaluru, Karnataka, India
}

*Corresponding Author:

Email: dramitay@gmail.com

\begin{abstract}
Introduction: H. pylorus is found in half the population of the world. Worldwide, H. pylorus is by far the most common aetiological agent for non self-limiting gastritis. Socioeconomic conditions in childhood is identified as a major risk factor for infection. Many studies have been conducted to establish the association of alcohol consumption and H Pylori gastritis, however the results reported by these studies were inconsistent.

Need of the present study is to assess the relation of alcohol consumption to H. pylori infection.

Objective: To assess the proportion of H. pylori and atrophic gastritis in alcoholic patients with upper gastrointestinal symptoms. Materials and Methods: A prospective study was conducted from October 2013 to June 2015 in the Department of Gastroenterology, M.S. Ramaiah Hospitals (M.S. Ramaiah Teaching Hospital and M.S. Ramaiah Memorial Hospital), Bangalore. 150 alcoholic patients were involved in the study. Age, sex, presenting complaints and detailed history regarding alcohol intake was taken. Rapid urease test was done for all the patients and a gastric biopsy sample was obtained from the antrum of the stomach and sent to the pathology department. Sections made from the gastric biopsy sample were stained with $\mathrm{H}$ and $\mathrm{E}$ and Giemsa stain. Sections were then evaluated for the presence of H. pylori.

Results: In the 150 alcoholic patients included in the study, prevalence of $H$. pylori was $76 \%$. The age and the gender did not play a role in the $\mathrm{H}$. pylori infection. It was observed in the study that the prevalence of $\mathrm{H}$. pylori infection increased with the increase in frequency, duration, amount of alcohol intake with a statistically significant $p$ value. It was found that the consumption of spirit was associated more with $\mathrm{H}$. pylori positivity than beer intake.

Conclusion: The prevalence of $\mathrm{H}$. pylori infection increases with the increase in frequency, duration and quantity of alcohol intake. The type of alcohol consumed also plays a role in $\mathrm{H}$. pylori infections.
\end{abstract}

Keywords: H. pylori, Alcohol intake, Gastric biopsy.

\section{Introduction}

Gastritis is an inflammatory condition of the gastric mucosa with different aetiologies, phenotypic expressions and natural histories. ${ }^{1,2}$ Worldwide, Helicobacter pylori (H. pylori) is by far the most common aetiological agent of non self-limiting gastritis. Longstanding inflammation of the gastric mucosa is thought to trigger a cascade of genotypic and phenotypic derangements that may eventually result in intestinal-type (or epidemic) gastric cancer (GC). ${ }^{3,4}$

It has been established that Helicobacter pylori $(\mathrm{H}$. pylori) infection is a major cause of gastric diseases around the world, including gastric and duodenal ulcer disease, and chronic gastritis. ${ }^{5} \mathrm{H}$ pylori has been shown to be associated with gastric malignancy, more commonly MALT type. ${ }^{7}$

Worldwide, the epidemiology of gastritis overlaps that of H.pylori infection which affects approximately $50 \%$ of the world's population. According to a study by Hunt RH et al, the prevalence of H.pylori in India is $88 \%$ and in South India $80 \%{ }^{8}$

Since there were inconsistencies in the published literature regarding association of $\mathrm{H}$ pylori infection and alcohol consumption, this study was planned with the aim to assess the proportion of H.pylori induced atrophic gastritis in alcoholic patients.

\section{Materials and Methods}

Source of Data: Gastric biopsies from alcoholic patients received for routine histopathological evaluation from the Department of Gastroenterology, M.S. Ramaiah Hospitals (M.S. Ramaiah Teaching Hospital and M.S. Ramaiah Memorial Hospital), Bangalore, from October 2013 to June 2015 were enrolled for the study.

Inclusion Criteria: Patients with history of alcohol intake presenting with upper gastrointestinal symptoms to M.S. Ramaiah Hospitals to Medicine/Gastroenterology Services and consenting for gastroduodenoscopy and biopsy were included in the study.

\section{Exclusion Criteria:}

1. Patient with other major gastrointestinal disorder

2. Patients who are not consenting for endoscopy.

3. Patients who are on anti-biotics.

4. Patients who had received anti H. Pylori therapy in the past 6 months.

5. Inadequate samples.

Method of Collection of Data: Age, sex and presenting complaints of the patients were noted. A detailed history was collected from each patient regarding the type, duration, frequency and amount of alcohol intake. Patients were categorized based on the main type of alcohol consumed like beer, wine and 
spirit. The frequency of alcohol intake was categorised as, 1 time monthly or less, 2-4 times monthly 2-3 times weekly and 4 or more times weekly. Alcohol intake was calculated in standard units/week, with 1 unit of alcohol equal to $30 \mathrm{ml}$ of whisky or $250 \mathrm{ml}$ of beer.

Written consent was taken from each patient and endoscopy was performed by the gastroenterologist and a gastric biopsy sample was collected from each patient from the antral mucosa. Apart from this, an additional biopsy sample was collected from each patient from the antrum, for rapid urease test (RUT).

Commercially available Pylo Dry kit was used to perform the rapid urease test on the gastric biopsy samples in all the patients. The change in the colour of the media of the Pylo Dry test kit from yellow to pink was taken as a positive test in all the cases.

The biopsy sample obtained for histological study was fixed in formalin $(10 \%)$ and submitted to the department of pathology in a separate container with proper labelling.

The gastric biopsy samples were processed and subsequently blocks were made. Two histology sections (5 micrometers thick) were obtained from each paraffin block. All the sections were stained with H\&E and Giemsa stain.

Microscopic examinations of the sections were carried out. Microscopically, in each case, presence of H. pylori was looked for, both in $\mathrm{H}$ and $\mathrm{E}$ stained sections and Giemsa stained sections. Giemsa staining of the sections were done for better identification of $\mathrm{H}$. pylori.

Finally proportion of $\mathrm{H}$. pylori was compared with the type, frequency and amount of alcohol intake.

\section{Statistical Analysis}

Descriptive statistics of $H$. pylori has been analysed and presented in terms of percentage and its 95\% confidence interval is estimated. Chi-Square test is used to compare the H. pylori proportion between age, sex, type, frequency and amount of alcohol intake.

\section{Results}

In the present study conducted in Pathology department of M.S. Ramaiah Medical College and Hospitals for a period of 21 months, from October 2013 to June 2015, 150 alcoholic patients who underwent gastric biopsy were included in the study.

The results obtained are as follows:

Table 1: Age and sex distribution

\begin{tabular}{|l|c|c|c|}
\hline \multirow{2}{*}{ Age (Years) } & \multicolumn{2}{|c|}{ Sex } & \multirow{2}{*}{ Total } \\
\cline { 2 - 3 } & Male & Female & Ten \\
\hline \multirow{2}{*}{$41-40$} & 48 & 0 & 48 \\
\cline { 2 - 4 } & $32.9 \%$ & $0.0 \%$ & $32.0 \%$ \\
\hline \multirow{2}{*}{$61-60$} & 92 & 1 & 93 \\
\cline { 2 - 4 } & $63.0 \%$ & $25.0 \%$ & $62.0 \%$ \\
\hline $61-80$ & 6 & 3 & 9 \\
\hline
\end{tabular}

\begin{tabular}{|l|c|c|c|}
\hline \multirow{3}{*}{ Total } & $4.1 \%$ & $75.0 \%$ & $6.0 \%$ \\
\cline { 2 - 4 } & 146 & 4 & 150 \\
\cline { 2 - 4 } & $100.0 \%$ & $100.0 \%$ & $100.0 \%$ \\
\hline
\end{tabular}

Age and Sex Distribution: Of the 150 cases, 146 were males and 4 were females. Maximum number of patients were in the age group of 41-60 years $(62.0 \%)$ and only $6 \%$ of the cases were between $61-80$ years of age. (Table 1)

Table 2: H. pylori positivity by rapid urease test

\begin{tabular}{|l|c|c|}
\hline $\begin{array}{c}\text { RUT (Rapid } \\
\text { urease test) }\end{array}$ & Number & Percent \\
\hline Negative & 36 & 24.0 \\
\hline Positive & 114 & 76.0 \\
\hline Total & 150 & 100.0 \\
\hline
\end{tabular}

$76 \%$ of the cases showed RUT positivity.

Table 3: H. pylori positivity in biopsy

\begin{tabular}{|l|c|c|}
\hline $\begin{array}{l}\text { Chronic Non Specific } \\
\text { Gastritis }\end{array}$ & Number & Percent \\
\hline $\begin{array}{l}\text { H Pylori Induced } \\
\text { Chronic Gastritis }\end{array}$ & 114 & 24.0 \\
\hline Total & 150 & 10.0 \\
\hline
\end{tabular}

$76 \%$ of the cases showed H Pylori positivity in biopsy.

Table 4: Frequency of alcohol intake and H. pylori infection

\begin{tabular}{|l|c|c|c|}
\hline \multirow{2}{*}{$\begin{array}{c}\text { Frequency of } \\
\text { alcohol intake }\end{array}$} & \multicolumn{2}{|c|}{ H. Pylori } & \multirow{2}{*}{ Total } \\
\cline { 2 - 4 } 2-4 times & Positive & Negative & Toth \\
monthly & 1 & 0 & 1 \\
\cline { 2 - 4 } $2-3$ times weekly & $100.0 \%$ & $0.0 \%$ & $100.0 \%$ \\
\cline { 2 - 4 } & $26.1 \%$ & $73.9 \%$ & $100.0 \%$ \\
\hline \multirow{2}{*}{$\begin{array}{l}\text { 4 or more times } \\
\text { weekly }\end{array}$} & 107 & 19 & 126 \\
\cline { 2 - 4 } Total & $84.9 \%$ & $15.1 \%$ & $100.0 \%$ \\
\hline & 114 & 36 & 150 \\
\cline { 2 - 4 } & $76.0 \%$ & $24.0 \%$ & $100.0 \%$ \\
\hline
\end{tabular}

Chi-square $34.36, \mathrm{p}$ value is $<0.001$.

Frequency of Alcohol Intake and $H$. pylori Infection: Of the 150 patients, 126 patients consumed alcohol 4 or more times weekly, of which 107 (84.9\%) were $\mathrm{H}$. pylori positive. 23 patients consumed alcohol 2-3 times weekly of which only $6(26.1 \%)$ were positive for H. pylori. Only 1 patient consumed alcohol 2-4 times monthly and he was $\mathrm{H}$. pylori positive. It was found that the number of $\mathrm{H}$. pylori positive cases increased with increase in the frequency of alcohol intake, which is statistically significant (Table 4). 
Table 5: Type of alcohol intake and $H$. pylori infection

\begin{tabular}{|l|c|c|c|}
\hline \multirow{2}{*}{ Type of alcohol. } & \multicolumn{2}{|c|}{ H. Pylori } & \multirow{2}{*}{ Total } \\
\cline { 2 - 4 } & Positive & Negative & Tecir \\
\cline { 2 - 4 } & 111 & 28 & 139 \\
\hline \multirow{2}{*}{ Beer } & $79.9 \%$ & $20.1 \%$ & $100.0 \%$ \\
\cline { 2 - 4 } & $27.3 \%$ & $72.7 \%$ & 11 \\
\hline \multirow{2}{*}{ Total } & 114 & 36 & $100.0 \%$ \\
\cline { 2 - 4 } & $76.0 \%$ & $24.0 \%$ & $100.0 \%$ \\
\hline
\end{tabular}

Chi-square $15.45, \mathrm{p}$ value is $<0.001$
Type of Alcohol Intake and H. pylori Infection: Of the 150 patients, 139 patients consumed spirit and only 11 patients consumed beer. Out of 139 patients who consumed spirit, 111 were $\mathrm{H}$. pylori positive $(79.9 \%)$ and $28(20.1 \%)$ were $\mathrm{H}$. pylori negative. Of the 11 patients who consumed beer, only $3(27.3 \%)$ were $\mathrm{H}$. pylori positive and $8(72.7 \%)$ were $\mathrm{H}$. pylori negative. Consumption of spirit was associated more with $\mathrm{H}$. pylori positivity, which is statistically significant (Table 5).

Table 6: Duration of alcohol consumption and H. pylori infection

\begin{tabular}{|l|c|c|c|}
\hline Duration of alcohol & \multicolumn{2}{|c|}{ H. pylori infection } & \multirow{2}{*}{ Total } \\
\cline { 2 - 3 } consumption in years & Positive & Negative & \\
\hline \multirow{2}{*}{$1-5$} & 21 & 9 & 30 \\
\cline { 2 - 4 } & $70.0 \%$ & $30.0 \%$ & $100.0 \%$ \\
\hline \multirow{2}{*}{$6-10$} & 57 & 24 & 81 \\
\cline { 2 - 4 } & $70.4 \%$ & $29.6 \%$ & $100.0 \%$ \\
\hline \multirow{2}{*}{$11-15$} & 21 & 1 & 22 \\
\hline \multirow{2}{*}{$>16$} & $95.5 \%$ & $4.5 \%$ & $100.0 \%$ \\
\hline \multirow{2}{*}{ Total } & 15 & 2 & 17 \\
\cline { 2 - 4 } & $88.2 \%$ & $11.8 \%$ & $100.0 \%$ \\
\cline { 2 - 4 } & 114 & 36 & 150 \\
\hline
\end{tabular}

Chi-square $7.9, \mathrm{p}$ value is 0.047

Duration of Alcohol Consumption and H. pylori Infection: Among 150 patients, 81 patients consumed alcohol for a period of 6-10 years. Of these 81 patients, H. pylori were positive in 57 patients (70.4\%). Only 17 patients consumed alcohol for more than 16years but among these 17 patients, 15 patients $(88.2 \%)$ were $\mathrm{H}$. pylori positive. Increase in the duration of alcohol consumption was associated with increase in $\mathrm{H}$. pylori positivity, which is statistically significant (Table 6).

Table 7: Units of alcohol consumption and H. pylori infection

\begin{tabular}{|l|c|c|c|}
\hline \multirow{2}{*}{$\begin{array}{c}\text { Units of alcohol } \\
\text { consumption/week }\end{array}$} & \multicolumn{2}{|c|}{ H. pylori infection } & \multirow{2}{*}{ Total } \\
\cline { 2 - 4 } & Positive & Negative & \\
\cline { 2 - 4 } & 2 & 12 & 14 \\
\hline \multirow{2}{*}{$15-28$} & $14.3 \%$ & $85.7 \%$ & $100.0 \%$ \\
\hline \multirow{2}{*}{$29-42$} & 41 & 15 & 56 \\
\cline { 2 - 4 } & $73.2 \%$ & $26.8 \%$ & $100.0 \%$ \\
\hline \multirow{2}{*}{$>43$} & 46 & 6 & 52 \\
\hline \multirow{2}{*}{ Total } & $88.5 \%$ & $11.5 \%$ & $100.0 \%$ \\
\cline { 2 - 4 } & 25 & 3 & 28 \\
\cline { 2 - 4 } & $89.3 \%$ & $10.7 \%$ & $100.0 \%$ \\
\cline { 2 - 4 } & 114 & 36 & 150 \\
\hline
\end{tabular}

Chi-square $36.6, \mathrm{p}$ value is $<0.001$

Units of Alcohol Consumption and H. pylori Infection: Of the 150 patients, 14 patients consumed alcohol between 1-14 units/week, of which only 2 (14.3\%) patients were $\mathrm{H}$. pylori positive. 28 patients consumed $>43$ units of alcohol/week, of which 25 (89.3\%) patients were positive for $\mathrm{H}$. pylori. As the units of alcohol consumption increased, the number of
H. pylori positive cases also increased, which is statistically significant.

\section{Discussion}

150 patients were involved in the present study of which 114 (76\%) tested positive for H. pylori infection, by both RUT test and biopsy. According to this study 
the prevalence of $\mathrm{H}$. pylori in alcoholic individuals is $76 \%$.

Age and Sex Distribution in the Study Group: It is observed in the study that $\mathrm{H}$. pylori positivity showed positive correlation with age of the patient from $68.8 \%$ to $88.9 \%$ but it was not statistically significant with a $p$ value of 0.284 .

Li Zhang et al conducted a study to investigate the association of $\mathrm{H}$. pylori with alcohol consumption in patients with functional dyspepsia. In their study the mean age was 45.8 years and the age range was $16-82$ years. The active $H$. pylori infection rate showed positive correlation with age from 20.8 to $35.7 \%$, but this was not statistically significant $(\mathrm{P}=0.67) .{ }^{9}$

According to our study, the association between sex and $H$. pylori infection was not statistically significant ( $\mathrm{p}$ value is 0.255 ). Li Zhang et al also did not observe any difference between $H$. pylori seropositivity and gender. $(\mathrm{P}=0.56){ }^{9}$

Age and sex of the patient did not play a role in $\mathrm{H}$. pylori positivity and was similar to the results obtained in the previous studies.

Relation of Alcohol Intake to $H$. pylori Infection: It is observed in the study that the number of $\mathrm{H}$. pylori positive cases increased with increase in the frequency of alcohol intake which is statistically significant.

Also the consumption of spirit is associated more with H. pylori positivity. This shows that the type of alcohol may have a role in the presence of $\mathrm{H}$. pylori and is consistent with the results obtained by Brenner et al which showed higher seropositivity in subjects consuming spirit as compared to wine and beer. ${ }^{10}$

There is a direct positive correlation between duration of alcohol consumption and units of alcohol consumption with $\mathrm{H}$ pylori infection. Li Zhang et al found that non-drinkers exhibited a significantly lower rate of $\mathrm{H}$. pylori infection (11.4\%) compared with drinkers $(32.7 \%)$, demonstrating a positive association between alcohol and $H$. pylori infection. They also revealed that the $\mathrm{H}$. pylori positivity rate appeared to increase with the amount of alcohol consumed ( $\mathrm{P}$ $=0.014) .{ }^{9}$ This was similar to the results obtained in the present study.

But studies done by Ogihara et al., 2000, Murray et al., 2002, Kuepper-Nybelenet al., 2005 have reported a significantly inverse association with $H$. pylori infection and alcohol intake. ${ }^{11-13}$

Few other studies have found no association (Rosenstock et al., 2000; Ito et al., 2001; Moayyedi et al., 2002). ${ }^{14-16}$

Brenner et al studied H. pylori seropostivity in 425 individuals and found that $50 \%$ of study population consumed beer, $46 \%$ consumed wine and small percentage consumes spirit. They reported that consumption of $75 \mathrm{~g}$ or less and more than $75 \mathrm{~g}$ alcohol per week was associated with a 37 and 66 percent reduction of the odds of $H$. pylori infection, respectively, compared with abstinence from alcohol ( $\mathrm{p}$ $=0.024){ }^{10}$

Studies from Germany (Brenner et al., 2001) and Greece (Gikas et al., 2004) suggest that an inverse relationship exists between alcohol consumption and $\mathrm{H}$. pylori infection that may be U-shaped rather than monotonic. Existence of a U-shaped relation might also explain why observed effects of alcohol consumption on $H$. pylori infection have been inconsistent in epidemiologic studies. ${ }^{17,18}$

Alcohol-induced disorders of the GI tract are very common. ${ }^{19}$ Both acute and chronic alcohol consumption can alter gastric acid secretion, induce acute gastric mucosal injury and interfere with gastric and intestinal motility. ${ }^{20}$ Studies in both animals and humans have found that alcohol concentrations of greater than $10 \%$ disrupt the gastric mucosal barrier and increase the mucosa's permeability. ${ }^{21}$ Several studies have suggested that a decrease in prostaglandins and an increase in the production of leukotrienes might play a role in the development of alcohol-induced mucosal injury. ${ }^{21,22}$ However, it appears that alcohol consumption is associated with active $\mathrm{H}$. pylori infection. It is postulated that alcohol consumption facilitates $H$. pylori infection by damaging the gastric mucosa. However, other mechanisms may be involved in the synergistic effect, including bacterial adherence and host factors. ${ }^{9}$

Bacterial adherence to the host epithelia is an important virulence determinant and it is an essential pre-requisite for pathogenicity for some bacteria. ${ }^{23}$ Bacteria with stronger adherence capacity are supposed to colonize with higher densities. ${ }^{24}$ The well-defined bacterial adherence factor, blood group Ag-binding adhesin (BabA) (llver et al., 1998), play a role in $\mathrm{H}$. pylori-induced severe gastric inflammation. ${ }^{25} \mathrm{H}$. pylori sialic acid-bindind adhesion has been implicated in persistent infection and chronic inflammation. ${ }^{26}$ The outer inflammatory protein, HP0638, is a novel putative virulence factor that is associated with interleukin -8 (IL-8) secretion from epithelial cells and ameliorates the adherence properties of $\mathrm{H}$. pylori and thereby augments the density of bacterial colonization. ${ }^{27,28}$

Briefly, acute and chronic alcohol consumptions disrupt the gastric mucosal barrier and increase the mucosa's permeability, resulting in a chemical inflammation. Subsequently, macrophages and neutrophils release the cytokine IL- 8 , which combines with its receptor in the endotheliocyte, and facilitate further development of the inflammation and also up regulate the expression of adhesion molecules such as inter-cellular adhesion molecule 1 (ICAM-1) and lymphocyte function-associated antigen-1 (LFA-1). IL8 may interact with HP0638 to augment the adherence capacity of $\mathrm{H}$. pylori and increase the colonization density. ${ }^{9}$ 
The above studies, explain the positive relationship of alcohol consumption to $\mathrm{H}$. pylori infection and atrophic gastritis.

\section{Conclusion}

The prevalence of $\mathrm{H}$. pylori infection increases with the increase in frequency, duration and quantity of alcohol intake. The type of alcohol consumed also plays a role in $\mathrm{H}$. pylori infections. There is a positive relationship of alcohol consumption to $\mathrm{H}$ pylori infection according to our present study.

\section{References}

1. Correa P. The epidemiology and pathogenesis of chronic gastritis: three etiologic entites. Journal of

Gastrointestinal Research 1980;6:98-08.

2. Graham DY. Helicobacter pylori infection in the pathogenesis of duodenal ulcer and gastric cancer: a model. Gastroenterology. 1997;113:1983-91.

3. Schistosomes, liver flukes and Helicobacter pylori. IARC Working Group on the Evaluation of Carcinogenic Risks to Humans. 1994;61:1-41.

4. Uemura N, Okamoto S, Yamamoto S. Helicobacter pylori infection and the development of gastric cancer. New England Journal of Medicine. 2001;345:784-9.

5. Kalantar J, Eslick GD, Talley NJ. Chronic gastritis and nonulcer dyspepsia. Current Topics in Microbiology and Immunology. 1999;241:31-5.

6. Talley NJ, Li Z. Helicobacter pylori: testing and treatment. Expert Rev Gastroenterology and Hepatology. 2007;1:71-9.

7. Eslick GD, Lim LL-Y, Byles JE. Association of Helicobacter pylori infection with gastric carcinoma: a meta-analysis. American Journal of Gastroenterology. 1999;94:2373-9.

8. Hunt RH, Xia SD, Megraud F, Leon-Barua R, Bazzori F, Vander Merwe $\mathrm{S}$ et al. Helicobacter pylori in Developing countries. World Gastroenterology Organisation Global guideline. Journal of Gastrointestinal and liver Disease. 2011;45:383-8.

9. Zhang, L, Guy D, Eslick, Harry H.-X. Xia, Chengqiu Wu, NghiPhung, Nicholas J. Talley. Relationship between alcohol consumption and active Helicobacter pylori infection. Alcohol and Alcoholism. 2010;45:89-94.

10. Brenner H, Rotherbacher D, Bode G, Adler G. Inverse graded relation between alcohol consumption and active infection with Helicobacter pylori. American Journal of Epidemiology. 1999;149:571-6.

11. Ogihara, A. Relationship between Helicobacter pylori infection and smoking and drinking habits. Journal of Gasteroenterology and Hepatology. 2000;15:271-6.

12. Murray LJ, Lane AJ, Harvey IM, Donovan JL, Nair P, Harvey RF. Inverse relationship between alcohol consumption and active Helicobacter infection: the Bristol Helicobacter project. American Journal of Gastroenterology. 2002;97:2750-5.

13. Kuepper-Nybelen J, W. Thefeld, D. Rothenbacher, Brenner H. An inverse relation between alcohol consumption and $\mathrm{H}$. pylori infection. Alimentary Pharmacology and Therapeutics 2005;21:57-64.

14. Rosenstock SJ, Jorgensen T, Andersen LP. Association of Helicobacter pylori infection with lifestyle, chronic disease, body indicesand age at menarche in Danish adults. Journal of Public Health. 2000;28:32-40.

15. Ito LS, Oba SM, Hamajima $\mathrm{N}$ et al. Helicobacter pylori seropositivity among 963 Japanese Brazilians according to sex, age, generation, and lifestyle factors. Japan Journal of Cancer Research. 2001;92:1150-6.

16. Moayyedi P, Axon ATR, Feltbower R. Relation of adult lifestyle and socio-economic factors to the prevalence of Helicobacter pylori infection. International Journal of Epidemiology. 2002;31:624-31.

17. Brenner H, Bode G, Adler G, Hoffmeister A, Koeniq W, Rothenbacher D. Alcohol as a gastric disinfectant? The complex relationship between alcohol consumption and current Helicobacter pylori infection. Epidemiology. 2001;12:209-14.

18. Gikas A, Triantafillidis JK, Apostolidis N et al. Relationship of smoking and coffee and alcohol consumption with seroconversion to Helicobacter pylori: a longitudinal study in hospital workers. Journal of Gastroenterology and Hepatology. 2004;19:927-33.

19. Riezzo G, Chiloiro M, Montanaro S. Protective effect of amtolmetinguacyl versus placebo diclofenac and misoprostol in healthy volunteers evaluated as gastric electrical activity in alcohol-induced stomach damage. Journal of Digestive Science. 2001;46:1797-804.

20. Bode C, Bode JC. Alcohol's role in gastrointestinal tract disorders. Alcohol Health Research World. 1997;21:76-3.

21. Bode JC, Bode C. Alcohol malnutrition and the gastrointestinal tract. Nutrition and Alcohol. 1992;48:403-28.

22. Bode C, Maute G, Bode JC. Prostaglandin E2 and prostaglandin $\mathrm{F} 2 \alpha$ biosynthesis in human gastric mucosa: effect of chronic alcohol misuse. Gastroenterology. 1996;39:348-52.

23. Jones GW. The attachment of bacteria to the surface of animal cells. Microbial Interaction. London: Chapman \& Hall. 1977; 139-76.

24. Rad R, Gerhard M, Lang R et al. The Helicobacter pylori blood group antigen-binding adhesin facilitates bacterial colonization and augments a nonspecific immune response. Journal of Immunology. 2002;168:3033-41.

25. Ilver D, Arnqvist A, Ogren. Helicobacter pylori adhesion binding fucosylatedhisto-blood group antigens revealed by retagging. Science. 1998;79:373-5.

26. Mahdavi J, Sonden B, Hurtig Met. Helicobacter pylori Sab Aadhesin in persistent infection and chronic inflammation. Science. 2002;97:573-8.

27. Yamaoka Y, Kwon DH, Graham DY. Proinflammatoryouter membrane protein (oipA) of Helicobacter pylori. National Academy of Science. 2000;97:7533-8.

28. Yamaoka Y, Kikuchi S, Zimaity HM. Importance of Helicobacter pylori oipA in clinical presentation, gastric inflammation, and mucosal interleukin 8 production. Gastroenterology. 2002;123:414-24.

How to cite this article: Rakshitha $\mathrm{HB}$, Amita K, Gouri M, Balekuduru A. Relation of alcohol to Helicobacter pylori infection: A cross sectional study. J Diagn Pathol Oncol. 2018;4(3):145-149. 